\title{
Marketing digital en micro y pequeñas empresas de publicidad de Bogotá
}

\author{
Clara Inés Uribe Beltrán* \\ Daniel Fernando Sabogal Neira**
}

Fecha de recibido: 25 de febrero de 2020

Fecha de aprobado: 30 de septiembre de 2020

Para citar este artículo: Uribe Beltrán, C. I., \& Sabogal Neira, D. F. (2021). Marketing digital en micro y pequeñas empresas de publicidad de Bogotá. Revista Universidad $\mathcal{E}$ Empresa, 23(40), 1-22. https://doi.org/10.12804/revistas.urosario.edu.co/empresa/a.8730

\section{Resumen}

Este artículo presenta los resultados de una investigación llevada a cabo en micro y pequeñas empresas de publicidad de Bogotá (Colombia). Su objetivo fue identificar las herramientas en línea usadas en sus estrategias de marketing digital, entre ellas el uso de las redes sociales y acciones de medios sociales. La metodología fue de tipo exploratorio-descriptivo, mediante la técnica de análisis de contenido, en una muestra estadísticamente representativa de 365 empresas. Se hicieron, además, 140 encuestas a empresarios como forma de contraste al análisis. Entre los resultados principales se encontró que la mayoría de micro y pequeñas empresas publicitarias aún no tienen plataformas de comercio electrónico ni realizan acciones de posicionamiento en buscadores. Las redes sociales más usadas son Facebook e Instagram, donde en mayor medida difunden imágenes y contenidos propios. En conclusión, las empresas de este

* Magíster en Comunicación Digital, Universidad Pontificia Bolivariana (Colombia). Especialista en Informática, Fundación Universitaria del Área Andina (Colombia). Especialista en Gerencia Estratégica de Mercadeo, Universidad Nacional Abierta y a Distancia (Colombia). Profesora de publicidad y mercadeo, Fundación Universitaria Los Libertadores (Colombia). Correo electrónico: ciuribeb@libertadores.edu.co

** Doctor en Comunicación Social, Universidad Nacional de Rosario (Argentina). Magíster en Docencia, Universidad de La Salle (Colombia). Profesional en Publicidad, Universidad Jorge Tadeo Lozano (Colombia). Profesor asociado II, Universidad Jorge Tadeo Lozano. Correo electrónico: danielf.sabogaln@utadeo.edu.co 
tamaño aún no usan de modo eficiente o estratégico los recursos digitales para su autopromoción. Se evidencia la necesidad de capacitación específica para los empresarios del sector, fortalecimiento en la representación gremial y se vislumbra una oportunidad para este tipo de empresas en relación con las economías creativas, si emprenden procesos de digitalización.

Palabras clave: marketing digital; publicidad; pymes; agencias; Bogotá.

\title{
Digital Marketing in Micro and Small Advertising Companies in Bogotá
}

\begin{abstract}
This article presents the results of an investigation carried out in micro and small advertising companies in BogotáColombia. The objective was to identify the online tools used in their digital marketing strategies, including the use of social networks and social network actions. The methodology used was exploratory-descriptive, using the content analysis technique performed on a statistically representative sample of 365 companies. In addition, 140 surveys were conducted on entrepreneurs as a way of contrasting the analysis. The main results show that the majority of micro and small advertising companies do not yet have an e-commerce platform or perform search engine positioning actions. The most used social networks are Facebook and Instagram, where, to a greater extent, they disseminate their own images and content. The research allowed concluding that companies of this size still do not make efficient or strategic use of digital resources for their self-promotion. The need for specific training for businesspeople in the sector is evident, as well as the reinforcement of the union and envisioning a relation with creatives with these typee of companies if digitalization processes are carried out.
\end{abstract}

Keywords: Digital marketing; advertising; agencies; Bogotá.

\section{Marketing digital em micro e pequenas empresas de publicidade de Bogotá}

\section{Resumo}

Este artigo apresenta resultados de uma investigação realizada em micro e pequenas empresas de publicidade de Bogotá - Colômbia. Objetivo: identificar as ferramentas online utilizadas em suas estratégias de marketing digital, entre elas o uso das redes sociais e ações de mídias sociais. Metodologia: tipo exploratório - descritivo, mediante técnica de análise de conteúdo realizada em uma amostra estatisticamente representativa de 365 empresas. Adicionalmente, realizaram-se 140 pesquisas de opinião com empresários, como forma de contraste à análise. Resultados principais: a maioria das micro e pequenas empresas publicitárias ainda não possuem plataforma de comércio eletrônico e não realizam ações de posicionamento em plataformas de busca. As redes sociais mais usadas são Facebook e Instagram, onde compartilham imagens e conteúdos próprios. Conclusões: as empresas deste tamanho ainda não fazem uso eficiente ou estratégico dos recursos digitais para autopromoção. Evidencia-se a necessidade de capacitação específica dos empresários do setor, fortalecimento da representação em conselho, e se vislumbra uma oportunidade para este tipo de empresas em relação às economias criativas, caso avancem em processos relativos ao meio digital.

Palavras-chave: marketing digital; publicidade; PMEs; agências; Bogotá. 


\section{Introducción}

El concepto de marketing ha evolucionado en las últimas décadas, desde la consolidación de internet, por lo cual se habla actualmente de un marketing "en lo digital", que tiene en cuenta, además de los criterios tradicionales, la integración de tecnologías asociadas a la evolución tecnológica y a la gestión de datos masivos. A su vez, la inversión publicitaria se enfoca progresivamente en canales en línea y así es como América Latina y Colombia han mostrado un aumento en el acceso y en el tiempo de conexión, disponibilidad de dispositivos y uso de los recursos que ofrece la red.

Colombia es uno de los países de la región donde en mayor medida se ha adoptado el marketing digital (Interactive Advertising Bureau [IAB], 2016), lo cual puede explicarse por el uso extendido del dispositivo móvil en el rango de los 24 a los 45 años (96\%) y a que ha sido uno de los mercados donde se ha integrado más rápidamente la actividad en línea, enfocada principalmente en redes sociales y en consultas para la compra de bienes y servicios (Deloitte, 2018). La ciudad de Bogotá concentra más del 50\% de esta actividad económica y, por esta razón, prontamente las empresas dedicadas a ofrecer servicios de publicidad vieron en las acciones de marketing a través de internet un valor agregado para sus clientes, nuevas líneas de negocio o una forma de alcanzar nuevos mercados.

Paralelamente, se ha observado un fenómeno de concentración global, alianzas y fusiones del negocio de la publicidad (Reig et al., 2012), producto del cual buena parte de la inversión publicitaria corresponde a anunciantes de marcas globales, que son atendidos por redes de agencias full service. Esta concentración deja un espacio para la atención de empresas de menor tamaño y, por esto, en América Latina, de manera similar a lo que sucede en diversos sectores de la economía, las pymes son mayoría en el renglón de la publicidad. En Colombia, tal como lo indican las cifras de registro de las empresas ante los entes reguladores, más del $95 \%$ del mercado publicitario está compuesto por micro y pequeñas empresas. La ciudad de Bogotá sigue esta tendencia.

Así, dentro del amplio sector de servicios, el subsector de las industrias creativas ha sido el de mayor expansión en Bogotá, con una concentración del $74 \%$ de las empresas pertenecientes a esta cadena de valor en el país, de las cuales el $55 \%$ son de las actividades 
publicidad y diseño (Invest in Bogotá, 2018), enmarcadas en la denominación genérica de publicidad. Este concepto engloba lo relacionado con la comunicación comercial tradicional, producción de material publicitario, marketing, producción audiovisual, relaciones públicas, branding, entre otros. Debido a esta amplia oferta de servicios, se ha dificultado distinguir entre los enfoques de cada grupo de empresas y, en consecuencia, la posibilidad de encaminar políticas públicas o programas de capacitación por sectores.

Como se explicará en la metodología, para la investigación que se está reportando, se tomaron las empresas que en su matrícula mercantil ante la Cámara de Comercio de Bogotá corresponden al código cilu 7310. Esta categoría distingue ocho actividades específicas, desde la asesoría en servicios creativos, creación de anuncios, representación en medios, entrega de material publicitario, alquiler de espacios, creación de estands o el manejo integral de campañas de mercadeo.

Otra problemática que se ha considerado como antecedente a este estudio es la representación gremial de la empresa publicitaria en relación con la micro y pequeña empresa. El triángulo tradicional del negocio publicitario (anunciantes, medios de comunicación y empresas de publicidad) está representado en Colombia por las siguientes asociaciones: la Unión Colombiana de Empresas Publicitarias, la Asociación Colombiana de Medios de Comunicación y la Asociación Nacional de Anunciantes. En el 2007, ingresó el IAB, con el fin atender la difusión y expansión de la publicidad interactiva, para lo cual agrupa, además de los actores del triángulo mencionado, a nivel local y multinacional, empresas de investigación, auditoría, servidores de anuncios (adservers) y proveedores de tecnologías.

Estas agremiaciones lideran e influyen la toma de decisiones del sector y algunas políticas públicas; pero sus estatutos derivan en cuotas de membresía que no están al alcance de las micro y pequeñas empresas, lo cual conduce a que no se vean directamente favorecidas por ese trabajo gremial. Así mismo, por razones similares, el acceso a decisiones, innovaciones tecnológicas, soporte y actualización de sus funcionarios también está fuera de su alcance. A la par que se puede ver como un problema, también debe asumirse como una oportunidad para la formalización y la digitalización de este sector, a la luz de nuevas leyes y políticas gubernamentales para el fomento de emprendedores de las industrias creativas. 
Esta investigación tuvo como objetivos identificar las herramientas digitales empleadas por las micro y pequeñas empresas de publicidad de Bogotá para su estrategia de marketing digital y determinar los medios sociales que actualmente están empleando, con miras a generar un conocimiento específico sobre el sector. Se partió de la hipótesis de que este grupo no está usando las herramientas digitales dentro de su estrategia y no emplean para su autopromoción los recursos que sí recomiendan a sus clientes. Con lo anterior se buscó generar una discusión sobre las oportunidades que aún está en capacidad de ofrecer la digitalización para el sector publicitario.

\section{Revisión de la literatura}

\subsection{Marketing digital y medios sociales}

Los cambios impulsados por la tecnología han conducido a replanteamientos, desde distintas esferas, de la forma como se ofrecen los servicios, se promocionan las empresas y se piensan las estrategias de marketing, involucrando los medios digitales. El concepto de marketing digital incorpora dos elementos: por un lado, el concepto de marketing y, por el otro, el de tecnología. El primero, según Kotler y Armstrong (2013), tiene el propósito de entender al cliente, para que el producto o servicio se ajuste tan bien a él que se venda solo. Kotler et al. (2016) agregan que el perfil de los nuevos consumidores da cuenta que el futuro del marketing tendrá una fusión entre experiencia en línea y fuera de línea, ya que "cuando decidan comprar, lo harán basados en la experiencia personal obtenida de la interfaz automatizada y de la interacción humana” (p. 15).

Ahora bien, Castells (1997) incluye dentro de las tecnologías de la información "el conjunto convergente de tecnologías de la microelectrónica, la informática (máquinas y software), las telecomunicaciones/televisión/radio y la optoelectrónica" (p. 60). De estos postulados, se desprende el concepto de marketing digital o marketing en lo digital, que, como afirman Kotler y Armstrong (2013), "es la forma de marketing con el más rápido crecimiento; actualmente, es difícil encontrar una empresa que no utilice la red de una manera significativa" (p. 27). 
Aclaremos este punto concentrándonos en lo expuesto por Selman (2017), quien definió el marketing digital como "el conjunto de estrategias de mercadeo que ocurren en el mundo online y buscan algún tipo de conversión por parte del usuario" (p. 5) y agrega: tiene entre sus ventajas medir de forma precisa y continua los resultados. Entre las estrategias de producto, servicio al cliente, promoción, distribución y branding en el marketing digital, se incluyen las redes sociales, las cuales a su vez hacen parte de los llamados medios sociales, basados en la web 2.0 que permiten la creación e intercambio de contenido generado por el usuario.

Adviértase, en efecto, que las técnicas de planificación estratégica son similares a las utilizadas en el marketing tradicional. De acuerdo con Vértice (2010, p. 16), estas consisten en definir el grupo objetivo - para el caso digital, el buyer person, objetivos y estrategias generales de marketing-, así como los criterios de posicionamiento y diferenciación. Además, estrategias y políticas de marketing mix, estrategia de atención y fidelización de clientes, presupuesto, indicadores de rendimiento o KPI, control y ajustes.

Otro aspecto que no se puede desconocer en el marketing digital es la presencia de la marca en los escenarios virtuales. Los consumidores o, mejor, las personas hacen parte de la quinta $P$, como lo mencionan algunos autores, ya que están conectados. Por ello, "debemos involucrarlos como parte de la historia de la marca: aprender, adaptar, crear y compartir contenidos con ellos" (Stalman, 2014, p. 22).

Al mismo tiempo, el marketing digital utiliza infinidad de herramientas para lograr sus objetivos: una de ellas es el diseño del sitio web o de una tienda virtual para dar a conocer sus servicios o vender en línea. Desde esta perspectiva, intervienen el Search Engine Optimization (SEO), el Search Engine Marketing (SEM) y el Social Media Marketing (sMm), cuyo objetivo principal es crear contenido de calidad y atraer lectores para compartir este contenido (Arias, 2013).

Algo parecido a lo tratado en el punto anterior ocurre con el trabajo de medios sociales, que debe ir de la mano con las estrategias de marketing, definiendo objetivos, a quién se va a dirigir, qué se va a comunicar, los medios sociales requeridos para tal efecto y la medición de resultados (Kuster, 2018). Los medios sociales, de acuerdo con su función, 
se pueden clasificar como directorios, de comunicación, sitios de comunidades y sitios para archivar o compartir.

Por otro lado, el estudio de Hubspot (2018) mostraba que las empresas presentan diferentes desafíos en marketing digital, como generar tráfico y leads, oportunidades de venta, retorno de la inversión, entre otros. Además, evidencia los desafíos de las empresas latinoamericanas en el área de ventas: en primer lugar, el de cerrar más negocios (70\%); en segundo lugar, el de comercializar en las redes sociales (40\%), y, en tercer lugar, el de mejorar la eficacia del embudo de ventas (39\%). En cuanto a medios sociales, en Colombia el alcance de estos medios está en el 83,8\% (Comscore, 2018).

El estudio del Content Marketing Institute (2018), elaborado a partir de 1947 encuestas con profesionales alrededor del mundo dice que el $56 \%$ de las empresas informaron que el área de mayor crecimiento ha sido la de creación de contenidos. El 70\% considera que son mucho más efectivos en sus estrategias de content marketing vs. lo que pasaba hace un año. El 93\% de las empresas reportaron que ahora tienen un serio compromiso en cuanto a marketing de contenidos se refiere.

Dicho estudio demuestra que entre los formatos de contenido de mayor crecimiento están: el escrito, incrementado en índices cercanos al $61 \%$ y el de formatos como audio y video, con un $64 \%$. Además, se confirma que los artículos en los blogs, los white papers y los estudios de casos son los mejores formatos del marketing de contenidos.

El estudio del Boston Consulting Group (2019) muestra que las empresas latinas emplean, en general, métodos más tradicionales y con menor sofisticación a los utilizados en mercados más avanzados para la medición y atribución. Únicamente, el $41 \%$ de las empresas afirma usar modelos de atribución, el $49 \%$ evalúa esfuerzos de marketing con métricas de rentabilidad y el $69 \%$ utiliza métodos simples como aquellos basados en last clicks, first clicks, descartando la importancia de acciones y canales relevantes en las etapas iniciales del embudo (awareness, interest, etc.).

En Chile se adelantó una investigación documental con el propósito de identificar el rol que las redes sociales desempeñan en los planes de marketing de pequeñas y medianas empresas de este país. Los resultados encontrados fueron: 
Las Redes Sociales son una herramienta muy poderosa que las empresas pueden utilizar para llegar a sus clientes con un costo menor que los medios tradicionales [...] Pequeñas y medianas empresas, con presupuestos limitados, deben buscar constantemente nuevas maneras de llegar a los usuarios y subculturas, de la forma más eficiente posible, y hoy, estas nuevas redes le dan la posibilidad de encontrar una solución frente a este problema. Deben además entender los distintos roles del marketing para así evaluar si es conveniente competir dentro de una Red Social. (Claro Correa, 2016, p. 56)

Por su parte, en Colombia, una investigación concerniente a explorar el uso de medios de comunicación en línea y redes sociales, realizada por Ortegón Cortázar y Ortegón Cortázar (2014), con 191 encuestas aplicadas a empresas micro, pequeñas y medianas de Bogotá, evidenció que "las empresas pueden mejorar la efectividad de sus programas de marketing con un mayor entendimiento de los recursos y herramientas tecnológicas libres o licenciadas, disponibles y adaptadas al interés de cada empresa" (p. 99).

Uno de los hallazgos representativos de esta investigación fueron que el $58 \%$ de los encuestados afirmó que la empresa utiliza Facebook; en esta red social se realizan promociones, lanzamientos, descuentos, concursos, entre otros. En segundo lugar, las empresas reportan utilizar Twitter, con 32\%, seguido de Google+, YouTube y blogs (Ortegón Cortázar \& Ortegón Cortázar, 2014).

Otro de los hallazgos es la relación entre el uso de Twitter por parte de las empresas y la actividad de la empresa (donde la actividad de servicios está principalmente asociada al uso de Twitter, y la actividad de comercio, con su no uso). Respecto de la disposición de la empresa de un sitio web, existe una relación con las variables de antigüedad de la empresa, tamaño, cantidad de empleados que utilizan computadores y cantidad de empleados que utilizan internet en el trabajo (Ortegón Cortázar \& Ortegón Cortázar, 2014).

En la investigación de Aguirre y Rozo (2017), realizada a pymes de Bogotá, con relación a las redes sociales, se encontró que "la herramienta digital más utilizada por las pymes son las redes sociales, pues ofrecen una gran variedad de formas para pautar, mediante la segmentación personalizada del cliente al cual se quiere dirigir" (2017, p. 36). Además, determinaron: 
La red social más importante para generar ventas en las pymes es Instagram; gracias a la amplia variedad de formatos que ofrece, publicación de fotos sencillas o en carrusel, historias y videos, se pueden atraer clientes potenciales interesados en la historia de la empresa y quienes posteriormente pueden transformar ese interés en una compra real. (p. 37)

Agregan que Facebook se utiliza para la promoción, para brindar información sobre la marca, para educar y para interactuar activamente con los usuarios; entre tanto, Instagram, para publicar fotos, videos e historias que duran 24 horas. Twitter, por otro lado, es una plataforma enfocada en la opinión y noticias, para el servicio al cliente, y YouTube está enfocada en la pauta (Aguirre \& Rozo, 2017).

\section{Metodología}

La investigación se llevó a cabo mediante un estudio exploratorio descriptivo, por cuanto buscaba determinar qué herramientas emplean para su estrategia de marketing digital las micro y pequeñas empresas de publicidad de Bogotá. Se enfoca en una actividad económica específica para describir su situación actual y obtener información de ese clúster en un momento determinado. Hubo un muestreo con representatividad estadística para aplicar las técnicas encuesta y análisis de contenido.

El análisis de contenido recoge los planteamientos y estudios realizados por Bardin (1996, citado en Andréu Abela, 2011), Macnamara (2005), Piñuel Raigada (2002) y Neuendorf (2017). Se refiere a interpretaciones de productos comunicativos con el objetivo de extraer indicadores cuantitativos y cualitativos que lleven a inferir conocimientos y a obtener datos relevantes sobre sus condiciones de producción (Piñuel Raigada, 2002).

Al ser el objetivo principal identificar las herramientas que emplean para su estrategia de marketing digital las micro y pequeñas empresas de publicidad de Bogotá, se fundamenta en la hipótesis de que probablemente sus acciones de marketing digital se realizan de manera informal o intuitiva, es decir, no existe una estrategia planificada o intencionada de hacer 
publicidad de sí mismas con las herramientas y alternativas digitales que tienen a disposición. El criterio de clasificación fueron las categorías temáticas asociadas a las variables de marketing digital y medios sociales. En primer lugar, se estableció el servicio publicitario que presta la empresa, entre las posibilidades que ofrece la inscripción en la actividad económica de la matrícula mercantil. En segundo lugar, se determinaron los canales de difusión de la empresa, si esta realiza posicionamiento orgánico o anuncios pagados. Se determinó igualmente la existencia de página web y se identificaron sus medios sociales.

El análisis de contenido llevó también a codificar las capturas de pantalla de la página web, blog o de cada uno de los medios sociales de la empresa. Además, se determinó quién o quiénes son los destinatarios de los mensajes, acciones o estrategias en los canales digitales y la utilización de medios sociales para complementar el servicio o atender requerimientos de sus clientes actuales o potenciales. Además, con el fin de hacer un contraste con lo hallado en el análisis de contenido, se realizaron encuestas compuestas por diecisiete preguntas, entre abiertas y de elección múltiple.

\subsection{Muestra y codificación}

La muestra representativa se calculó por medio de un muestreo aleatorio simple, con una confiabilidad del 95\%. De un total de 7114 micro y pequeñas empresas matriculadas en Bogotá bajo la actividad económica publicidad, la muestra calculada fue de 365 empresas. De acuerdo con Tamayo (2011), "los procedimientos para seleccionar una muestra tienen como objetivo la mayor seguridad o probabilidad de que la muestra reproduzca las características de la población" (p. 180). Paralelamente, se realizaron 140 encuestas a empresarios de la publicidad, de forma aleatoria, no necesariamente correspondientes con las analizadas, a fin de comparar los resultados del análisis y conocer las respuestas de los empresarios de la publicidad respecto a los temas de la investigación. Además, para resolver algunas incógnitas que solo se resuelven con la técnica de la encuesta.

En cuanto a la codificación para el análisis de contenido, se determinaron doce códigos, surgidos de las variables del marketing digital y del smm, además de información para caracterizar las empresas. El código 1 define la actividad económica de la empresa. El 2 establece sus canales digitales de difusión (página web, redes sociales, mensajería instantánea, etc.). El código 3 especifica las redes sociales. El código 4 analiza los destinatarios 
de las acciones digitales de cada empresa. El 5 define si la empresa realiza acciones de posicionamiento en buscadores. El código 6 dice cuáles son los canales digitales por los cuales cada empresa realiza procesos de servicio al cliente. El 7 registra las redes sociales utilizadas para esos procesos de servicio al cliente. El código 8 consigna las estrategias de precio implementadas por las empresas en canales digitales. El 9 está orientado a determinar los procesos de distribución de servicios a través de canales digitales. El código 10 establece el tipo de contenido difundido por la empresa. El 11 determina acciones de publicidad nativa (no intrusiva). Finalmente, en el código 12 se buscaron las estrategias promocionales que las empresas llevan a cabo a través de herramientas digitales.

\section{Resultados}

De acuerdo con la actividad económica declarada en el registro mercantil de las empresas analizadas (código 1: tipo de servicio publicitario), los servicios que más declaran las empresas en su objeto social es la actividad de "asesoría, servicios creativos, producción de material publicitario y utilización de los medios de difusión", con un $50.9 \%$ de las empresas de la muestra. Para "Creación y colocación de anuncios en periódicos, revistas, programas de radio, televisión, internet y otros medios de difusión”, se ubica en un 19.7\%, y para "Creación y colocación de anuncios de publicidad exterior, mediante carteles, carteleras, tableros, decoración de vitrinas, diseño de salas de exhibición, colocación de anuncios en automóviles y autobuses, entre otros", en un 17.9\%.

Las anteriores cifras dan una idea general de las actividades que reconocen y practican en este tamaño de compañías y se hace evidente que todavía no se ha extendido la actividad relacionada con publicidad digital.

En el código 2, o canales de difusión, se reportan los siguientes indicadores significativos: un $83.9 \%$ de las empresas analizadas tiene página web sin plataforma de comercio

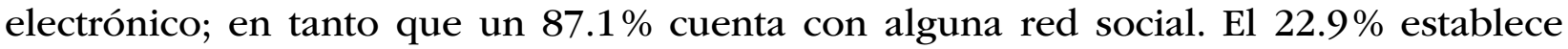
comunicación a través de mensajería instantánea (WhatsApp). El blog se usa en el 19\% de los casos. Las encuestas corroboran esta proporción, donde el 83.3\% de los empresarios 
manifiesta que ofrecen página web informativa sobre los servicios que presta la empresa, lo cual puede apreciarse en la figura 1.

3a. Si tiene página web, señale de qué tipo:

12 responses

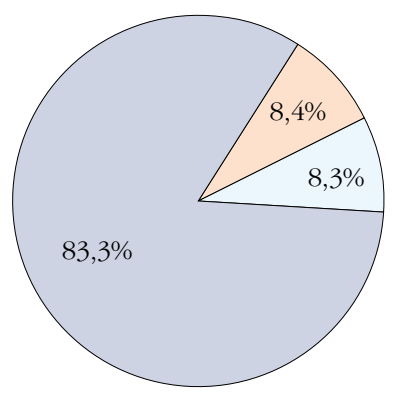

Informativa (solo informa los servicios que de su empresa)

Comercio electrónico (pagan y descargan por allí los servicios)

El usuario puede completar un formulario de contacto solicitando una orden de compra

Figura 1. Tipo de página web

Fuente: elaboración propia (2019).

Para las redes sociales indagadas en el código 3, observamos predominio de Facebook (82\%), seguida de Instagram (48.9\%), LinkedIn (38.5\%), Twitter (39.6\%) y YouTube (26.3\%). En esa misma categoría se incluyó a WhatsApp, con un $20.1 \%$. En cuanto a lo reportado por los empresarios en las encuestas, la tendencia (Facebook, Instagram, LinkedIn, Twitter) se mantiene con algunas variaciones en los porcentajes, como puede apreciarse en la figura 2.

10b. Si marcó la opción "Redes sociales" en la pregunta anterior ¿cuáles utiliza?

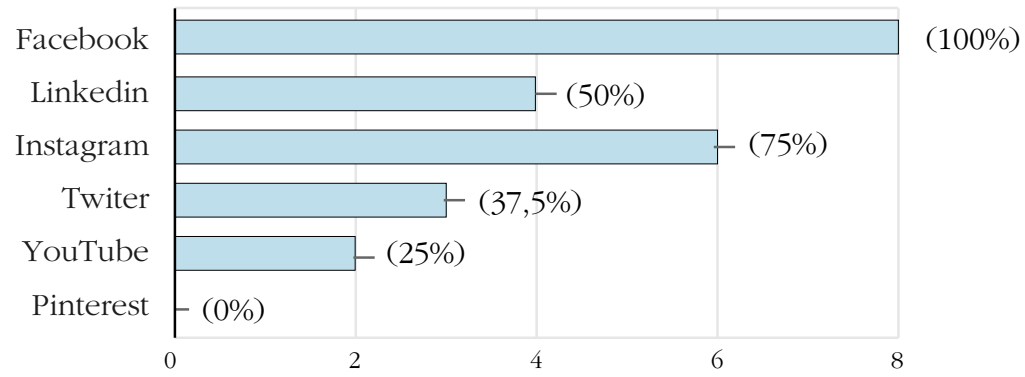

Figura 2. Canales de difusión de las micro y pequeñas empresas de publicidad Fuente: elaboración propia (2019).

En cuanto al código 4, donde se determinó quién o quiénes son los destinatarios de los mensajes, acciones o estrategias en los canales digitales de la empresa, se infiere por el 
tono y los argumentos empleados que la comunicación está dirigida a gerentes de empresas (70.6\%), luego a directores de marketing (65.6\%), público en general (26.3\%) y un $14 \%$ a grupos objetivos o nichos específicos. Las respuestas de la encuesta a empresarios sobre este aspecto coinciden con el análisis de contenido y guardan concordancia con la estructura de negocios para este tipo y tamaño de empresas, como se puede observar en la figura 3.

7. ¿Quién espera que vea los mensajes, acciones o estrategias en los canales digitales de su empresa? Marque todas las que considere.

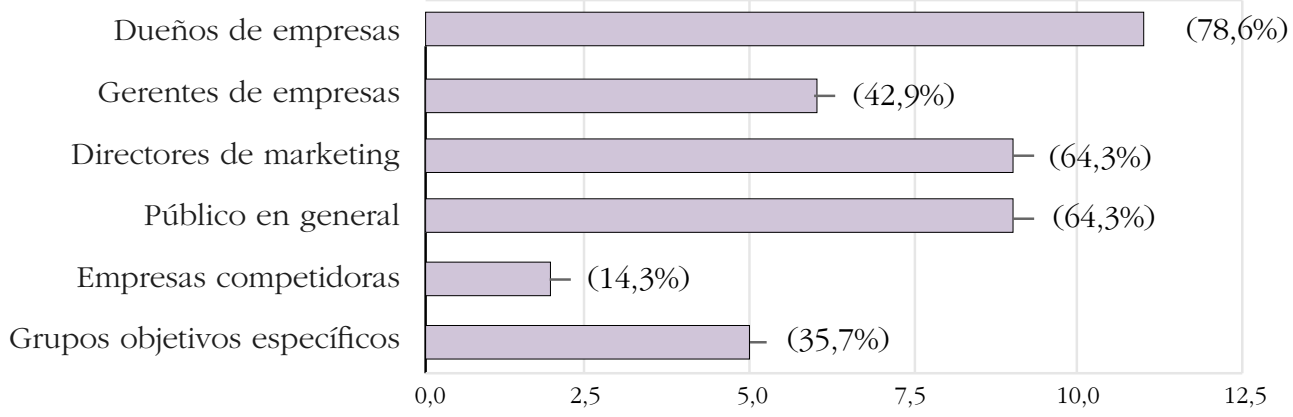

Figura 3. Grupo objetivo de las micro y pequeñas empresas de publicidad

Fuente: elaboración propia (2019).

En el rastreo de acciones de posicionamiento en buscadores, la verificación en cada una de las 365 empresas que conformaban la muestra establece que el $86.4 \%$ no realiza ninguna acción. El porcentaje minoritario que sí las realiza, lo hace a través de posicionamiento orgánico SEO o no pago.

El factor servicio al cliente (código 6) verificó si las empresas utilizan canales en línea para complementar el servicio o atender requerimientos de sus clientes actuales o potenciales, del cual se obtuvo un $\mathbf{9 5 . 3 \%}$ afirmativo. Los canales más usados fueron, en su orden, correo electrónico (82.5\%), formulario en la página web $(76.2 \%)$, chat de redes sociales (63.2\%), WhatsApp (26\%) y comentarios dentro de redes sociales (12.3\%). En porcentajes minoritarios se reportan Chatbot, Skype y área privada de clientes en la página web. La figura 4, tomada del análisis de contenido, muestra los canales preferidos para entrega de resultados de los servicios que prestan las empresas. Dentro de las redes sociales más usadas para servicio al cliente (código 7) se ubican Facebook (92.5\%), Instagram (30.2\%), LinkedIn (19.8\%) y Twitter (14.2\%). 


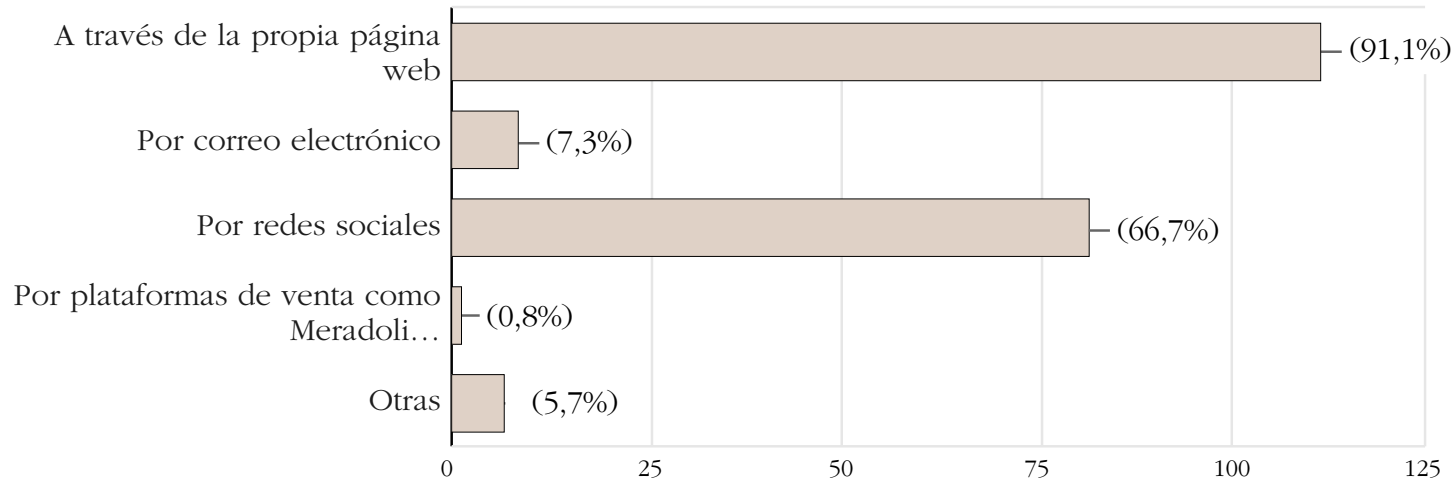

Figura 4. Canales preferidos para entrega de resultados de los servicios Fuente: elaboración propia (2019).

Pasando al factor precio (código 8), se comprobó si la empresa utiliza alguna estrategia de precio especial o descuento a sus clientes por contratar los servicios de su empresa a través de sus canales digitales. Un $\mathbf{9 7 . 5 \% ~ n o ~ r e a l i z a ~ n i n g u n a . ~ C a s o s ~ a i s l a d o s ~ i n f o r m a n ~}$ alguna estrategia de precio especial para fomentar una primera compra o descuento por pago en línea.

El factor de distribución (código 9) comprobó si la empresa emplea algún canal digital para entregar al cliente el resultado de los servicios publicitarios que vende. En este aspecto, se llega a un $52.8 \%$ que sí lo hacen, repartidos entre la propia página web (87.4\%), por redes sociales (63.6\%) y a través de correo electrónico (11.3\%).

El tipo de contenido que publican las micro y pequeñas empresas de servicios creativos para dar a conocer en sus canales digitales los servicios que prestan (código 10) muestra que lo más usado son las fotografías propias (86.2\%), seguido por los videos propios (58.2\%), las infografías (31.3\%) y los audios de elaboración propia (2.9\%).

Por último, dentro de las estrategias promocionales (código 11), se indaga por acciones de publicidad nativa no intrusiva, con una respuesta negativa del $95.8 \%$. A su vez, la búsqueda de otras estrategias de marketing digital que usa la empresa para promover sus servicios se destaca el contenido de marca (57.6\%), marketing de contenidos (52\%), ninguna (19\%) y comunidades (13.7\%), aunque en un porcentaje bajo se reporta la utilización de influencers para generar conversación acerca de sus servicios. Solamente en 
casos muy específicos se detectaron webinarios, eventos en línea, transmisiones en vivo o realidad aumentada. La figura 5 da cuenta de la totalidad de lo encontrado.

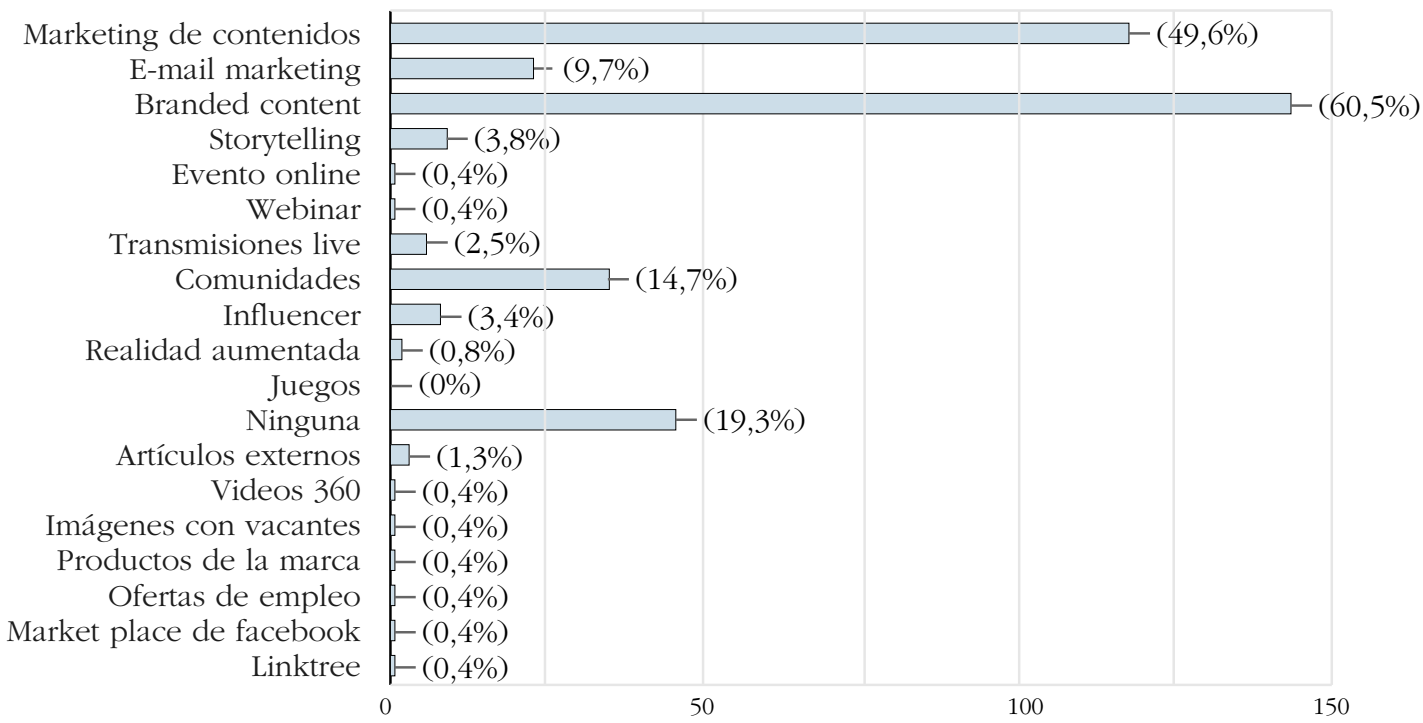

Figura 5. Estrategias de atracción de las micro y pequeñas empresas de publicidad Fuente: elaboración propia (2019).

Tal como se dijo en la metodología, se consideró necesario contrastar el análisis de contenido con encuestas a empresarios, para obtener información sobre algunos aspectos que el equipo investigador no estaba en condiciones de verificar directamente. Estos temas buscaron establecer certezas sobre estrategias de marketing para los entornos digitales, presupuesto, planes de acción a largo plazo, métricas, etc.

Entre las métricas más usadas para medir los resultados de las acciones de marketing digital sobresalen: tráfico, ventas y costo por adquisición, de acuerdo con las respuestas de la encuesta; mientras que en los medios sociales las métricas principales son: alcance, engagement y me gusta. Así lo resume la pregunta de la encuesta que se expone en la figura 6. 
17. ¿Cuál de las siguientes métricas de marketing digital tiene en cuenta con mayor frecuencia?

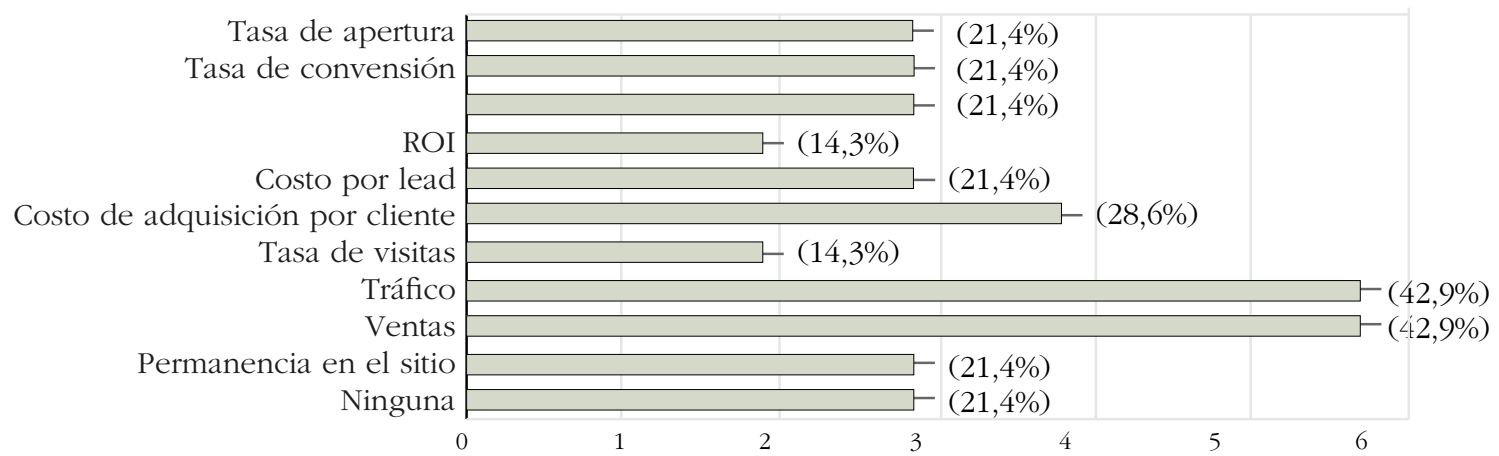

Figura 6. Métricas de marketing digital usadas con mayor frecuencia

Fuente: elaboración propia (2019).

Se pudo evidenciar, además, que el presupuesto mayormente asignado para las estrategias de marketing digital es del 0\% al 10\% del total (64,3\%). Donde más interés tienen los micro y pequeños empresarios de la publicidad en fortalecer su estrategia de marketing digital es en la formulación de la estrategia y herramientas de pauta $(37,5 \%)$ y en el desarrollo de acciones de marketing digital (21\%).

Al preguntarles a los empresarios si han elaborado un plan de acción a largo plazo para promover la presencia de la empresa en internet, el 57\% respondió sí, y el 42\%, no. Sin embargo, como lo podemos apreciar más adelante, tal parece que lo realizan a corto plazo y con acciones no planeadas dentro de una estrategia de marketing digital. Además, el objetivo principal es comercial, con un 42,9\% (figura 7 ).

2b. ¿Cuál es el objetivo principal de su plan o estrategia?

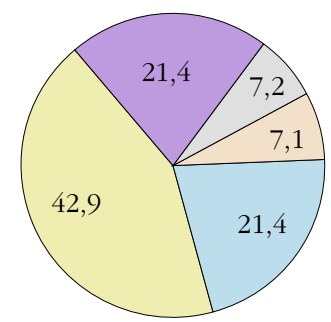

Objetivos de relaciones (crear, mantener e intensificar relaciones con clientes, proveedores, etc.)

Objetivos comerciales (aumentar, mantener ventas, cuota de mercado, cualquier objetivo de las 4 Pes)

Objetivos económicos (incrementar o mantener beneficios, rentabilidad...

Todos los anteriores juntos

No elabora estrategia

Figura 7. Objetivo principal del plan o estrategia Fuente: elaboración propia (2019). 
Las encuestas arrojan que los clientes aumentaron gracias a los medios digitales, muy a pesar de que como se evidencia no utilizan en un $100 \%$ el potencial del marketing digital y los medios sociales (figura 8).

2b. ¿Cuál es el objetivo principal de su plan o estrategia?

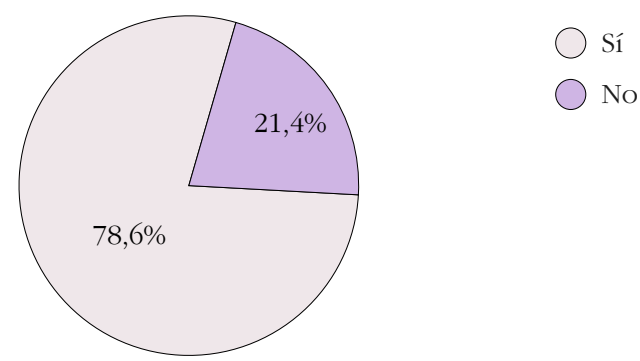

Figura 8. Aumento de clientes o ventas gracias a los medios digitales

Fuente: elaboración propia (2019).

\section{Discusión}

En los resultados principales de esta investigación se observa una baja adopción de los recursos del marketing digital en las empresas objeto del estudio, así como y un uso limitado de redes sociales y de las posibilidades de los medios sociales. Esto lleva a confirmar la hipótesis de que la micro y pequeña empresa de publicidad de Bogotá aún tiene mucho por incorporar y aplicar en sus estrategias.

Las cifras de diversos estudios, que muestran avances en acceso, inversión publicitaria y comercio electrónico, a la vez que en las empresas estudiadas hay baja adopción del marketing digital. En esto último coincide con investigaciones realizadas por el Ministerio de Tecnologías de la Información y las Comunicaciones de Colombia, Deloitte y el Observatorio de e-Commerce. También hay coincidencias referidas a que los esfuerzos de las pequeñas empresas se enfocan principalmente en redes sociales, según otro estudio realizado para todo el sector de servicios creativos (Ministerio de Tecnologías de la Información y las Comunicaciones, 2018). 
Los datos obtenidos son un aporte a la caracterización de las industrias culturales y creativas en Bogotá, que han venido realizando entidades como la Alcaldía de Bogotá y la Cámara de Comercio. Hasta el 2019, mostraba datos en el sector de artes escénicas y espectáculos artísticos, audiovisual y radio, artes visuales, música, libros y publicaciones; pero aún no se había caracterizado las micro y pequeñas empresas del sector publicitario de la ciudad. Otra contribución adicional se dirige a dar elementos para el fortalecimiento de la política pública distrital de economía cultural y creativa, bajo las directrices emitidas por la Unesco en el marco de las industrias culturales y creativas. Los servicios de las empresas publicitarias se incluyen en el grupo de tres grandes sectores: herencia cultural, creatividad y creaciones funcionales. Estas últimas, a su vez, han sido clasificadas en siete grupos: patrimonio, presentaciones artísticas, artes visuales, artesanía, libros y prensa, medios audiovisuales y servicios creativos (Unesco, 2013). Es decir, así como se muestra un panorama del marketing digital en un sector de la publicidad de Bogotá, esta investigación se da a conocer cuándo están surgiendo nuevas pautas para comprender y regular la pequeña y mediana empresa.

También se deben tener en cuenta las implicaciones en el enfoque de políticas públicas que recogen las anteriores reflexiones y clasificaciones. En el caso colombiano, están constituidas en la Ley 1834 de 2017, conocida como Ley Naranja, la cual abarca sectores relacionados con el emprendimiento creativo y "tiene como objeto desarrollar, fomentar, incentivar y proteger las industrias creativas. Estas serán entendidas como aquellas industrias que generan valor en razón de sus bienes y servicios, los cuales se fundamentan en la propiedad intelectual". Así es como, con respecto a la ciudad de Bogotá, datos de este estudio pueden contribuir con acciones de gobierno local como la Política de Economía Cultural y Creativa, que hace parte del Plan Distrital de Desarrollo 2016-2020 (Alcaldía Mayor de Bogotá, 2016).

Las limitaciones de este estudio tienen que ver con la clasificación de la actividad económica, a la luz de lo que se declara en la inscripción de la empresa a la Cámara de Comercio. Se considera que no hay suficiente flexibilidad y actualización de lo que significa hacer publicidad en los tiempos actuales. Algunas empresas pueden estar dedicándose a otra actividad, pero se ven forzadas a hacer su matrícula mercantil en la categoría genérica o más cercana a su objeto social.

Quedan pendientes, en primer lugar, estudios comparativos con las grandes agencias publicitarias o las agencias netamente digitales, cuyas mediciones no proceden generalmente 
de artículos académicos. Así mismo, una línea de trabajo puede surgir del debate sobre la representación gremial. Es probable que muchas políticas, teorías, modelos de negocio y organizaciones gremiales se estén viendo desde la gran empresa local o multinacional; los casos de éxito o aprendizajes de las grandes corporaciones no siempre van a encajar o funcionar con solvencia en estructuras tan pequeñas, que en algunos casos llegan a lo unipersonal.

Las acciones de marketing digital para la autopromoción de la micro y pequeña empresa de publicidad están más que todo orientadas a la consecución de nuevos clientes, pero también este estudio puede proponer el escenario posterior de la relación con cliente-agencia. Aquí es donde debería pensarse en capacitación y tecnología para nuevos desarrollos en marketing digital, medios sociales, entrega de servicios a través de plataformas digitales, pagos en línea, entre muchos otros. Es recomendable una mayor articulación con instituciones públicas y privadas que apoyan el emprendimiento, la formalización y la internacionalización en el sector estudiado.

\section{Conclusiones}

Las herramientas digitales empleadas por las micro y pequeñas empresas de publicidad de Bogotá para su promoción y consecución de clientes muestran la red social Facebook como la más usada; pero no se evidencia un aprovechamiento de todos sus recursos ni su papel dentro una estrategia definida de marketing digital. Además, utilizan principalmente el SEO para promover su página web en buscadores y los empresarios reconocen que en esas acciones no hay una intención estratégica y que deben reforzar sus conocimientos sobre estos aspectos.

En cuanto a la actividad económica, la mayoría de las empresas de este tamaño se dedican a variadas actividades relacionadas con los servicios creativos de publicidad. Se hace necesario que las entidades donde hacen su registro mercantil ayuden a crear categorías más específicas que incluyan nuevas necesidades del mercado y nuevas tecnologías. Esto puede ayudar a mejorar la caracterización de las empresas y el diseño de políticas y planes a la medida de este sector tan representativo. 
Ante esta baja adopción del marketing digital, los esfuerzos principalmente se enfocan en redes sociales, y esto es algo en lo que se coincide con un estudio realizado por el Ministerio de Tecnologías (2018) para todo el sector de servicio creativo. En relación con las investigaciones revisadas para este trabajo, se encuentran algunas coincidencias generales en cuanto al uso de redes sociales; una de ellas es la realizada por Aguirre y Rozo (2009), que reportan Facebook e Instagram como las dos redes sociales más usadas. Facebook es también la red de mayor uso en el estudio de Ortegón Cortázar \& Ortegón Cortázar (2014).

Por otro lado, se presenta una paradoja, por cuanto en el mercado actual, las empresas de servicios publicitarios deben enfocarse en ofrecer a sus clientes estrategias y desarrollos centrados en la digitalización y en el comercio electrónico; mientras que para promoverse a sí mismas aún recurren a métodos y prácticas tradicionales (tarjetas de contacto, llamadas telefónicas, visita a prospectos, recomendaciones de allegados). Por esta causa, se dejan de lado recursos de automatización, gestión de datos masivos y puesta en marcha de campañas digitales que podrían mejorarles sus indicadores.

En el campo específico de las redes sociales, queda en evidencia que no existe por parte de las empresas estrategia ni seguimiento. Los perfiles se abren, pero no se alimentan con contenido ni se da respuesta oportuna a los contactos que hacen clientes potenciales. Si, sumado a lo anterior, se evalúan las acciones de smM, se encuentra un bajo nivel de uso de sus recursos, restringido a casos aislados. Más que todo en las compañías que prestan servicios de publicidad tradicional o producción de avisos o acciones ligadas a los puntos de venta, no se evidencia inversión en acciones de posicionamiento en buscadores. Contrasta con agencias netamente orientadas a los servicios digitales, que se convierten en excepciones en este estudio.

Así, el grado de madurez digital de este tipo de empresas en la escala propuesta por CGB es "naciente", es decir, "usan datos de terceros y compra directa de medios, con baja vinculación y trazabilidad de la relación directa con las ventas del negocio" (Boston Consulting Group, 2019). Pocas empresas de la muestra llegan a la madurez digital emergente.

Adicionalmente, se justifica la insistencia en que el marketing en lo digital se planifique de manera integral, orientado hacia objetivos de negocio. Las métricas deben evaluarse y, 
así mismo, involucrar en lo digital todos los factores de marketing posibles, incluyendo diseño del producto, precio, distribución, ventas y posicionamiento.

\section{Referencias}

Aguirre, J. C., \& Rozo, J. D. (2017). Marketing digital en las pymes de Bogotá [tesis de grado]. Colegio de Estudios Superiores de Administración. https://repository.cesa.edu.co/bitstream/handle/10726/1816/TG_817.pdf?sequence=1\&isAllowed=y

Alcaldía Mayor de Bogotá. (2016). Plan de desarrollo distrital 2016-2020. http://www.saludcapital.gov.co/Documents/Transparencia/PDD_BMPT_2016_2020_Tomo_1.pdf

Andréu Abela, J. (2011). Técnicas de análisis de contenido: una revisión actualizada. Centro de Estudios Andaluces. https://www.centrodeestudiosandaluces.es/publicaciones/tecnicas-de-analisis-de-contenido-una-revision-actualizada

Arias, M. (2013). Marketing digital: posicionamiento SEO, SEM y redes sociales. IT Campus Academy.

Boston Consulting Group. (2019). Estudio data-driven marketing. http://image-src.bcg.com/ Images/Latin-America-onset-digital-maturity_tcm9-216643.pdf

Castells, M. (1997). La sociedad red. Alianza.

Claro Correa, D. (2016). El rol de las redes sociales en marketing de pequeñas y medianas empresas [tesis de pregrado]. Universidad de Chile. http://repositorio.uchile.cl/handle/2250/138870

Comscore. (2018, 13 de abril). Estado de social media en América Latina 2018. https://www. comscore.com/lat/Prensa-y-Eventos/Presentaciones-y-libros-blancos/2018/Estado-deSocial-Media-en-America-Latina-2018

Content Marketing Institute. (2018). Estudio del content marketing institute. https://www. wearecontent.com/blog/marketing-de-contenidos/estudio-del-content-marketing-institute

Deloitte. (2018). Consumo móvil en Colombia. https://www2.deloitte.com/co/es/pages/technology-media-and-telecommunications/articles/consumo-movil-2018.html

Hubspot. (2018). Estado del Inbound en Latinoamérica. http://www.stateofinbound.com/ america-latina-es

IAB Colombia. (2016, 3 de abril). Resumen ejecutivo del reporte de inversión de año 2016 acumulado. https://www.iabcolombia.com/resumen-ejecutivo-del-reporte-inversion-del-ano-2016-acumulado-2/ 
Invest in Bogotá. (2018, abril). Bogotá un centro global de negocios y un excelente lugar para vivir. https://es.investinbogota.org/sites/default/files/2018-05/Anexo\%201.pdf

Kotler, P., \& Armstrong, G. (2013). Fundamentos del marketing. Pearson.

Kotler, P., Kartajaya, H., \& Setiawan, I. (2016). Marketing 4.0: transforma tu estrategia para atraer al consumidor digital. LID.

Kuster, I. (2013). Marketing en la nueva era. Ibergarceta.

Ley 1834. (2017, 13 de mayo). Por medio de la cual se fomenta la economía creativa Ley Naranja. https://dapre.presidencia.gov.co/normativa/normativa/LEY\%201834\%20DEL\%20 23\%20DE\%20MAYO\%20DE\%202017.pdf

MacNamara, J. (2005). Media content analysis: Its uses, benefits and best practice methodology. Asia Pacific Public Relations Journal, 6(1), 1-34.

Ministerio de Tecnologías de la Información y las Comunicaciones. (2018, 26 de enero). Caracterización de las MiPyme colombianas y conocimiento de su relación con las Tecnologías de la información y las Comunicaciones-TIC. https://colombiatic.mintic.gov. co/679/articles-56356_recurso_1.pdf

Neundorf, K. (2017) The content analysis guidebook (2. ${ }^{\mathrm{a}}$ ed.). Sage.

Ortegón Cortázar, L., \& Ortegón Cortázar, L. (enero de 2014). Características de la micro, pequeña y mediana empresa asociadas a los tipos de tecnologías de comunicación y uso de medios sociales. Poliantea, 10(18), 85-110. https://dialnet.unirioja.es/servlet/articulo? codigo $=4916772$

Piñuel Raigada, J. L. (2002). Epistemología, metodología y técnicas del análisis de contenido. Estudios de Sociolingüística, 3(1), 1-42. https://www.ucm.es/data/cont/docs/268-2013-0729-Pinuel_Raigada_AnalisisContenido_2002_EstudiosSociolinguisticaUVigo.pdf

Reig, R., Mancinas Chávez, R., \& De Araujo Rodríguez, A. J. (2012). Concentración global del negocio de la publicidad. Los cuatro principales grupos: wPP, Omnicom group, Interpublic y Publicis. Global Media Journal, 9(18), 35-51. https://www.redalyc.org/articulo.oa?id=687/68724519003

Selman, H. (2017). Marketing digital. Ibukk.

Stalman, A. (2014). Brandoffon: El branding del futuro. Gestión.

Tamayo, M. (2011). El proceso de la investigación científica. Limusa.

Unesco. (2013). Políticas para la creatividad: Guía para el desarrollo de las industrias culturales y creativas. https://es.unesco.org/creativity/sites/creativity/files/220384s.pdf

Vértice. (2010). Marketing digital. Fundación Vértice. 